\title{
Experiences on Development and Design of STACK Problems for Circuit Analysis
}

\author{
By Otto Ellonen ${ }^{*}$, Maarit Vesapuisto ${ }^{ \pm} \&$ Timo Vekara ${ }^{*}$
}

STACK (System for Teaching and Assessment using a Computer Algebra Kernel) is an open-source assessment system for mathematics and related fields. At University of Vaasa, STACK has been used in courses of Mathematics and now it has been implemented in Electrical Engineering. This paper discusses experiences on development and design of various Circuit Analysis problems in STACK environment including student feedback. The starting point for developing problems here is that they do not include much calculation; instead the problems focus more on the representation of equations. The problems are designed to provide essential visual assistance to the students by drawing pictures, such as circuit diagrams by using JSXGraph library that is supported by STACK. With the help of JSXGraph, designer can draw various geometrical figures according to IEC 60617 standard. One important function in STACK is that it immediately gives automated feedback to the students depending on their answers. For this reason, a so-called potential response tree (PRT) is created for every problem. The PRT usually consists of several nodes, which compare whether specified conditions are fulfilled. The PRT can further be developed into a more complex one, which examines not only frequent but even rare errors made by students. These assets and learning tools seem to create a powerful basis for interactive learning process, where visual aids are used in solving Circuit Analysis problems in Moodle.

Keywords: STACK, JSXGraph, interactive learning, circuit analysis, IEC 60617.

\section{Introduction}

STACK is an open-source assessment system for mathematics and related fields created by Chris Sangwin in University of Birmingham in early 2000s (Sangwin 2013). The key concept of this system is to provide tools for creating mathematical Moodle-based questions which can generate automatic feedback for the students depending on their given answer (Moodle 2020). By using Maxima, STACK can evaluate the given mathematical input (Maxima 2020). STACK also supports graphical presentation of problems by utilizing JSXGraph JavaScript library.

The goal of this paper is to examine the development process of STACK problems in the field of Circuit Analysis at University of Vaasa. The purpose of these problems is to help the students to understand the most basic and

\footnotetext{
*Research Assistant, School of Technology and Innovations, University of Vaasa, Finland. ${ }^{ \pm}$Lecturer, School of Technology and Innovations, University of Vaasa, Finland.

tProfessor, School of Technology and Innovations, University of Vaasa, Finland.
} 
fundamental parts of each given topic in the field. With the help of these questions in addition to the course material, the students are able to solve conventional exercises more successfully.

\section{Background}

Circuit Analysis is one of the most fundamental aspects in electrical engineering. University of Vaasa has co-operation with Aalto University, where professors Erkki Voipio and Martti Valtonen created during tens of years sound basis on teaching circuit analysis exactly (Voipio 2006, Valtonen 2020). In Vaasa our teaching continues this tradition and circuit theory is currently divided into two courses: Circuit Analysis A and B from which the first one is held for the first-year students in the spring and the second one is held at the beginning of the $2^{\text {nd }}$ year in Autumn (University of Vaasa 2020). In order to successfully pass the Circuit Analysis B, the student must have acquired the fundamental skills and knowledge from the previous course which deals with the basics of DC and AC circuits. During the first year the students also attend the basic calculus course which is a prerequisite for the Circuit Analysis A and is organized during the first semester.

The Circuit Analysis A is often held up as one of the most challenging courses by the students during the first year due to number of different reasons. One of the reasons, which has been identified, by lecturer Maarit Vesapuisto is the applying of the mathematical knowledge when forming equations related to different circuit laws. It has also been noticed that some students do not draw the complete circuit diagrams with the needed arrows at all which makes the evaluation of their solution impossible. Others might get an answer that can possibly be right, but instead of thinking about it, they just move to the next problem. Another difficulty occurs when the students start experimenting with AC circuits and must start calculating with phasors and complex numbers. As stated in Neitola's study (2019), the University of Oulu has had similar problems when teaching circuit analysis (Neitola 2019).

The problems might also relate to understanding the concepts related to electrical circuits (McDermott and Schaffer 1992). For example, the students have difficulties in understanding the differences between current and voltage or how either of them work. Even when the students could mathematically solve the given problem, they would struggle with the answer when asked about the functionality of the circuit. This leads to another problem where the students can solve complex systems and models mathematically but fail to analyze the results they get. This can be problematic later in the studies and working life.

It should be noted that problems related to conceptual understanding of the basics that occur during the Circuit Analysis A should be dealt with during the course. Any misinterpret pieces of information are carried on to the Circuit Analysis B and will cause unwanted obstacles for the students which would make the passing of the course harder.

It has also been suggested that lab exercises can be used to motivate students when it comes down to learning about circuit analysis (Trajković 2011). The 
practical work can be an eye-opening experience in some cases where the students have been struggling to understand some of the concepts and might also find it more 'interesting' than the conventional lectures and exercises. As stated in Fino (2018) the problems arise with the increase in numbers of students attending the course.

Another way of improving the student's chances in learning circuit analysis in addition to traditional lectures and exercises is the implementation of simulation exercises. There are many different simulation programs like LTspice (Analog Devices 2020), APLAC (Cadence 2020) or MATLAB (Attia 1995) which can be used to enhance the students learning capabilities in circuit analysis. Student's skills to create equations and exact drawings for circuits are essential.

There also exist numerous helpful applications such as circuit calculators (WolframAlpha 2020) or circuit building and simulation tools like CircuitLab (CircuitLab 2020) or EveryCircuit (EveryCircuit 2020). These simulation tools let the students to play with circuits interactively. Different components and their values can be changed during runtime and the application generates visual help like animations and plots for the user to see how the changes in values and circuit affect the other properties like currents. Generally in circuit analysis, there are trends towards animations, simulations and interaction, still keeping on visuality with precise graphical drawings.

At University of Vaasa the Circuit Analysis courses include lectures with animations, weekly exercises and simulation exercises based on APLAC. In addition, from 2019 onwards STACK has been used during the course Circuit Analysis A.

\section{Developing Circuit Analysis Problems in STACK}

In this section a more in-depth look is given on developing exercises in the field of circuit theory. Earlier STACK has been used in the University of Vaasa in mathematics (linear algebra lectured by Matti Laaksonen) and based on the flexibility and accessibility it was chosen as the main development tool for the circuit analysis as well. The idea was to create numerous different and simplified problems that the students could perform and train on before trying to solve the more complex exercises. Another important objective was to focus more on what type of answers the students would have to give. In most cases the correct answer was an equation instead of a numerical value i.e., no calculation was needed. This is also the case particularly in questions regarding DC circuits in steady state as the calculation would be trivial and easily done with a calculator if the equation is right. For example, the most basic question would ask the student the voltage equation of a given one current loop with a voltage source and three resistors based on Kirchhoff's laws as seen in Figure 1. According to Kirchhoff's voltage law the sum of voltages around any closed loop is zero so the correct answer to the problem in that case would be

$$
E+U_{1}-U_{2}+U_{3}=0
$$


or

$$
-E-U_{1}+U_{2}-U_{3}=0 \text {. }
$$

Both answers are accepted as correct, and it does not matter which one is given. Note that since beginning of the studies it is essential to use symbols and figures based on international standards (Vesapuisto 2004).

Figure 1. Simple STACK Question used in University of Vaasa

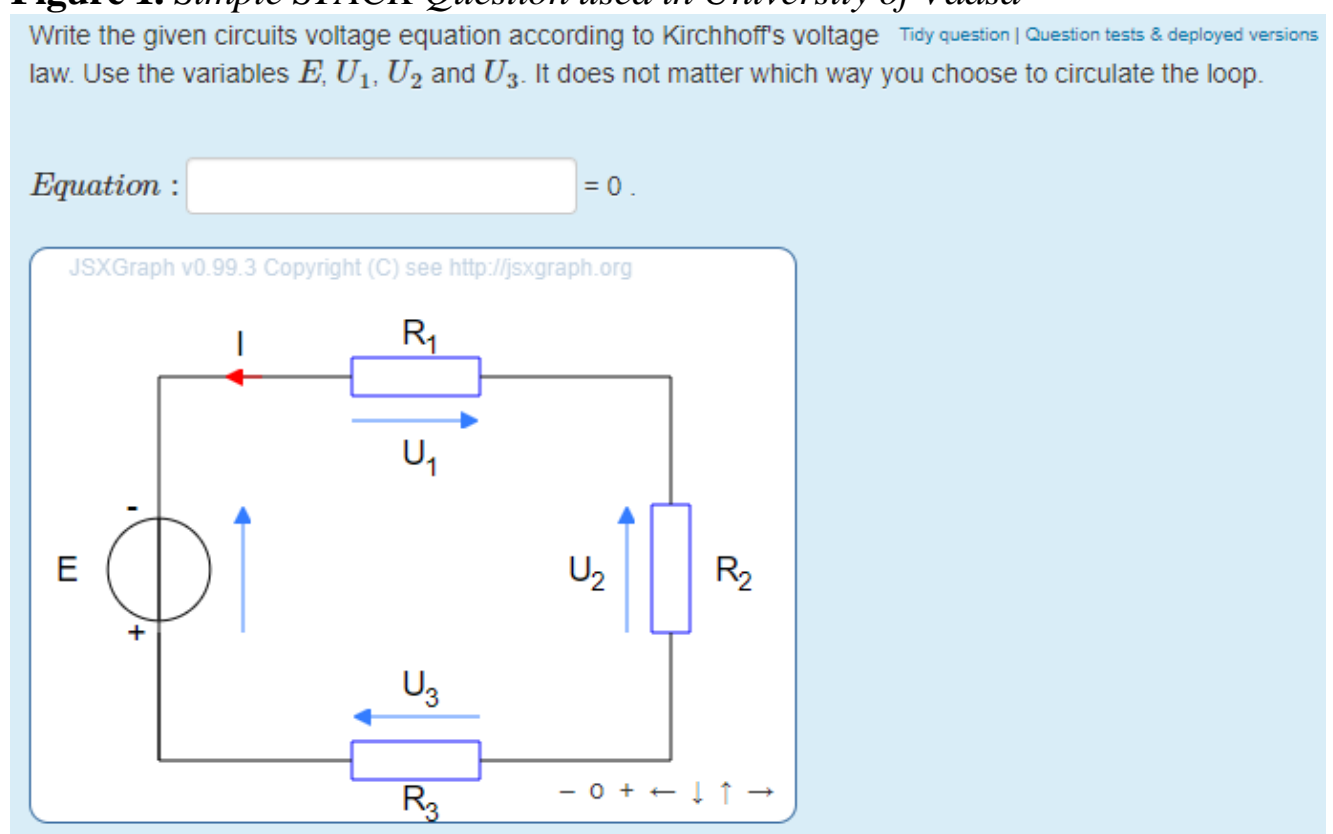

Source: Circuit Analysis A (University of Vaasa 2020).

\section{Design and Structure of a STACK Question}

The whole development process of the question includes many different stages before it can be presented to the students. The process consists of following main steps:

- Outlining the needs for the question type.

- Creation of the STACK question including PRT.

- Implementation.

The first step when creating a question for Circuit Analysis A is to address the different challenges the students might have with the learning process of the topics introduced during the course e.g., Kirchhoff's laws, mesh and nodal analysis, phasors (AC) etc. In order to learn the fundamentals of these topics, the questions (in addition to other lecture materials) should help the students understand the concepts better and boost their ability to solve the more complex exercises while at the same time understanding what they are actually doing. This can be achieved in many ways as described in the following sections. 


\section{Creation of a STACK Question in General}

In this section a general STACK question generation process is briefly summarized for the case of simplicity. The structure of a STACK question can be divided into following three main parts as described by Sangwin (2013):

- Question Variables.

- Question Text.

- Potential Response Tree.

The question variables contain different variables defined by the creator. These variables can be integers, functions or any expression which can be used when creating the logic for the question. For example, we could have a very simple question that asks for the sum of two different variables $x$ and $y$. The correct answer variable TAns would then be defined as the sum of $x$ and $y$ demonstrated in Figure 2. The variable TAns is used later in the Potential Response Tree to validate if the student answered correctly. The variables $x$ and $y$ will be shown to the students in the question text so they can answer the question correctly.

Figure 2. Example of Question Variables

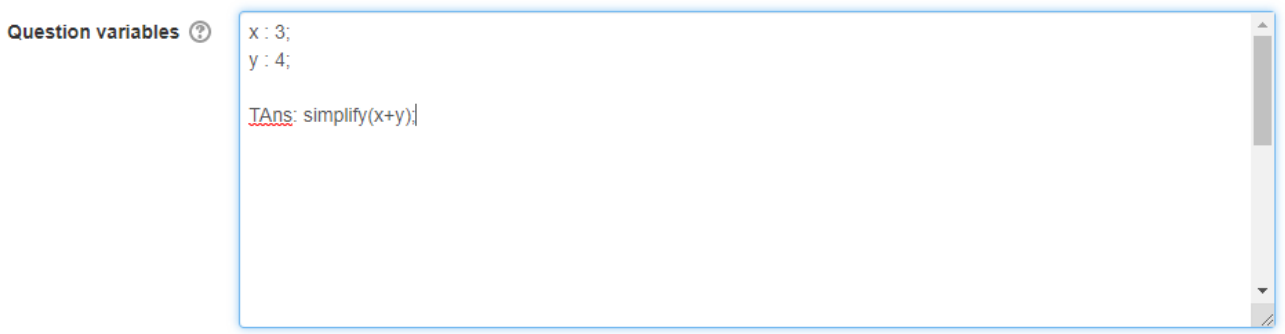

In the above example, the variables $x$ and $y$ get the values of 3 and 4 so the variable TAns will get the value of 7 .

The question text is considered as the main part of the question. This part includes all the instructions and information the student needs in order to solve the problem (e.g., assignment, student input fields, pictures etc.). The variables defined in question variables can be presented for the students in order to give a proper assignment for the problem. Considering the problem stated earlier, the student would be asked for the sum of $x$ and $y$. This could be formulated very simply to look like something presented in Figure 3. 
Figure 3. A Simple Question Text presented for the Student

The white box in Figure 3 is the input field where the student can write the answer. The input field types can be defined as algebraic input (used in the example), matrix, single character, a text area or a simple true or false dropdown answer. The question text is written in HTML where LaTeX and CAS (Computer Algebra System) commands can be embedded (STACK Documentation 2018).

The potential response tree (PRT) is the logical key element when giving feedback for the student. Here the student's input can be evaluated and compared using different kinds of tests. For example, an algebraic equivalent test can be used for comparing if the students answer is the same as the right answer. The response tree contains at least one node, but usually more are created in order to perform more tests. The tests are done within the nodes. Since the results of the tests can be either true (green) or false (red), the nodes have two outputs which can be connected to other nodes creating a 'tree-like' structure. Depending on the answer and the results of the tests, points can be given for the students and that helps to keep track on the progression. In Figure 4, a basic PRT consisting of two nodes is shown.

Figure 4. A Basic PRT Consisting of Two Testing Nodes connected to Each Other

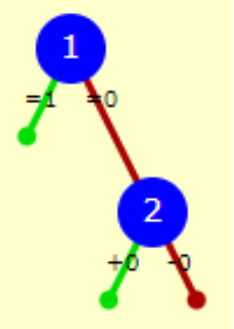

As stated above, the green line from the node is executed if the test result is true. In this case the first node awards one point if the students answer and the test answer are equal. Otherwise the red line is executed, no points are given, and the second test is performed in the next node, using the previous examples, the first node tests if the student has given the sum correctly. In case the answer is false, a 
second test is used to further analyze what kind of an error was made. For example, the second test checks if the student used subtraction instead of addition. The ans 1 mentioned is the input variable of the student answer.

The most important feature of the response tree is the ability to generate feedback depending on answers given by the students. In addition to grading, verbal and visual feedback can also be included. The most common feedback is telling the student if the answer was correct or not. With simple problems like the example above this short feedback might be enough. However, with more complex problems this might not be the case. Because of this, performing more tests with the students answer makes it possible to give more versatile and precise feedback which helps the student to realize what was wrong.

\section{Usage of JSXGraph in Circuit Analysis}

The visualization of a problem is a crucial part when solving questions related to circuit analysis since the problems, without few exceptions, always deal with a given circuit. As stated earlier with the use of pre-defined pictures e.g. circuit diagrams as components in the question text, it is possible to construct number of arbitrary circuit problems for the students. However, there are also possibilities to generate more sophisticated visual aids in the question.

Figure 5. Creating an Electrical Component with JSXGraph
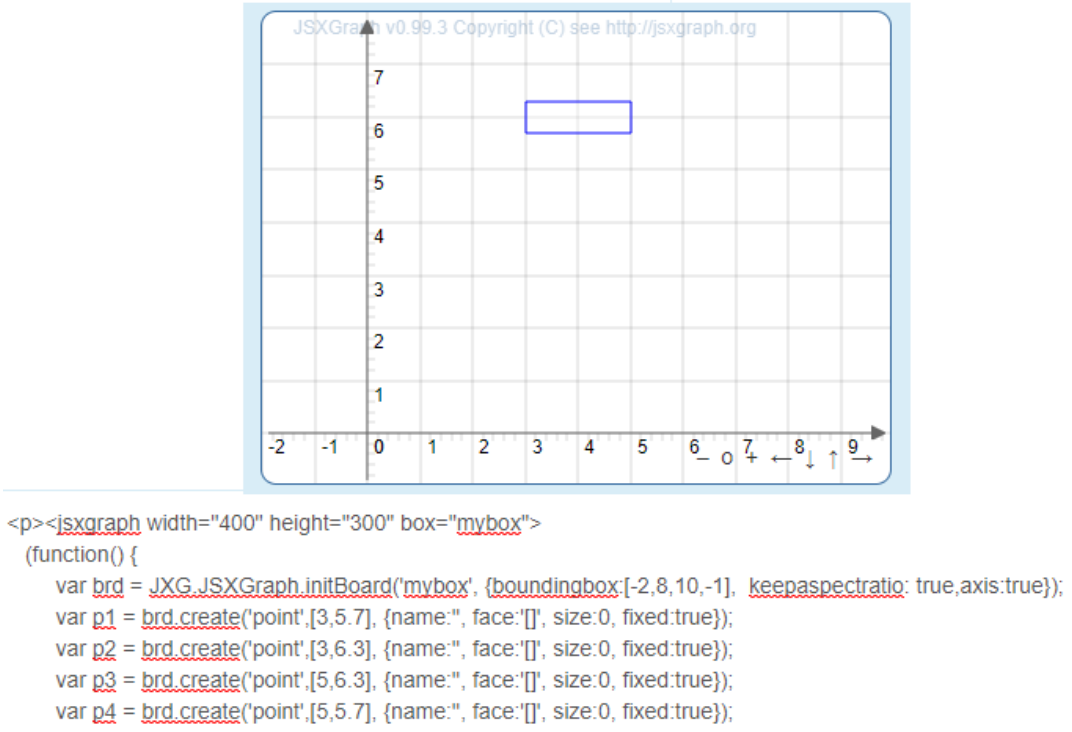

JSXGraph is a JavaScript library which is supported by STACK itself (JSXGraph 2020). It is a tool which enables the use of interactive geometry, plotting of functions and data visualization. The creator can draw geometrical 
figures such as lines, circles, boxes, etc., on a user-defined coordinate system. These figures are then generated when the student begins to solve the question. Usually these are used in mathematics with problems related to geometry, but they can also be manipulated to be used in other fields like circuit analysis. For example, different electrical components can be generated with the use of these geometrical figures. Consider a resistor which is shaped like a rectangle. There are many ways to achieve this with one requiring the creator to define the corners of the rectangle and using the 'polyon' element of JSXGraph which creates a rectangle according to the coordinates of the corners. In Figure 5 the above example with the related code is presented.

The coordinate system is printed inside the JSXGraph box whose dimensions are defined first. The variable $b r d$ is then created to hold the information regarding the board. Then four different points $\mathrm{p}_{1}-\mathrm{p}_{4}$ are created with different coordinates to describe the wanted component corners. Finally, the 'polygon' element with the previous parameters is created. In addition, one could make use of the 'text' element to display the name of the component i.e., $R_{1}$.

With the help of the various elements from the JSXGraph library also other electrical components can be created. With the 'line' element the generated components can be connected to each other visualizing wiring to make a proper looking circuit shown in Figure 1. The color of the drawn elements can also be changed to provide more visually pleasing outcome and to make it more distinct for the students. At University of Vaasa the current arrow is usually denoted with red and arrows used to imply voltages are blue as seen in Figure 1.

\section{Randomization of the Circuit}

To properly test the students' knowledge on circuit theory it should be tested with multiple unique situations and not the same ones repeatedly. On the other hand, it would need much work to make multiple versions of the same circuit manually with small changes and then implementing them later. For this purpose, there is an easy solution to this: randomization of the parameters. Different parameters and variables defined in question variables can also be randomized. As stated, before these values can be used later in the question text section including in the different JSXGraph elements. This means we can generate random components in selected positions. In Figure 6 two different situations of the same question are presented but with randomly generated components. This means that when the student attempts the quiz one of the two situations is picked. This can be achieved with a simple if-else statement. In this case a variable is defined in question variables which can have two different values chosen randomly when the code is run. The if statement simply checks which one was chosen and according to that a component is generated. This procedure can be used for every other component in the circuit which further adds more different versions. 
Figure 6. Two Versions of the Same Question generated when the Student Attempts the Quiz. Resistor is generated (a) and Voltage Source is generated (b) on the Right-Hand Side of the Circuit

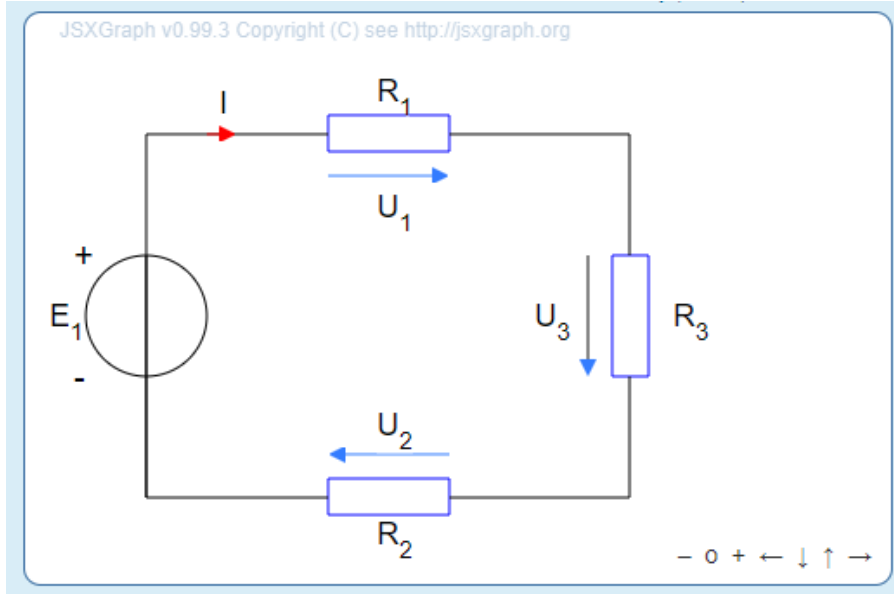

(a)

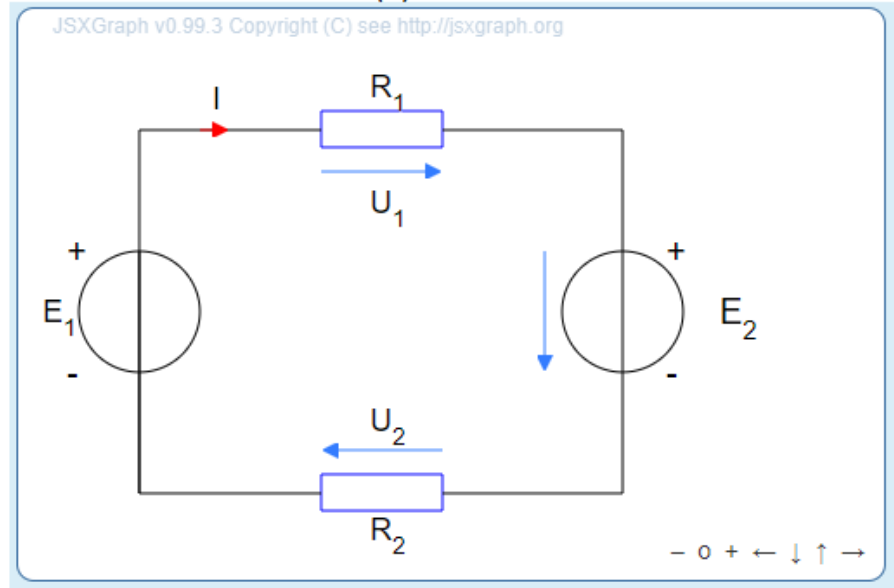

(b)

In Figure 6a, a resistor is generated on a selected place on the right-hand side of the circuit. In Figure $6 \mathrm{~b}$ a voltage source is generated instead. In such a trivial circuit changing one component with another does not really change the outcome or the equation that much so changing other things must be included.

The randomly generated values for the variables can also be included inside the elements of the JSXGraph. Most of the drawn elements must include some coordinates in order to properly draw the element in its wanted position. These coordinates can either be static numbers defined when creating the code or they can be replaced with variables which can be randomly generated when the code is run. For example, this allows us to randomly generate the directions of the voltage and current arrows in a circuit. Or we can rotate the voltage sources by randomly generating the coordinates for the poles. With these changes even the most trivial circuit can have multiple different versions and it becomes harder for the students to just memorize or guess the correct answers forcing them to solve the given task 
accordingly. With additional loops and more complex circuits, the blind guessing becomes almost impossible due to the big number of changing variables.

Adding randomness can be an efficient way of generating multiple versions of the same circuit. However, this also makes the modelling of the correct answer for the circuit a bit trickier. Manually inputting every possible answer and then determine which is correct for the given circuit would simply take too much time and in case of errors troubleshooting could get tedious. By our experience, a very simple method can be used to generate the model answer for each input. As stated before, the placement, rotation or direction of the elements can be generated randomly. The voltage arrow $U_{1}$ in Figure 6 could have two different directions: from left to right (as seen in the figure) or from right to left. Considering the situation of Figure 6a, the circuits voltage equation would be

or

$$
E_{1}-U_{1}-U_{2}-U_{3}=0
$$

$$
-E_{1}+U_{1}+U_{2}+U_{3}=0
$$

If we were to change the direction of $U_{1}$ to go from right to left the above equations would change to

or

$$
E_{1}+U_{1}-U_{2}-U_{3}=0
$$

$$
-E_{1}-U_{1}+U_{2}+U_{3}=0 \text {. }
$$

We can see that the only thing that changes is the sign before $U_{1}$. This leads us to a very simple solution of the model answer. Each of these signs are based on the directions of the voltage arrows in the circuit and since the directions are defined with randomized variables, we can use them in the model answer as factors to define the correct polarization of the variables. In Figure 7, a value for the variable ' $\mathrm{s} 1$ ' is defined from a set of two numbers: -1 and 1 . The variables ' $\mathrm{x} 1$ ' and ' $\mathrm{x} 2$ ' are then used to define the $\mathrm{x}$-coordinates of the start and end point of the voltage arrows with the help of the randomized variable. The arrow is a 'line' element which has an attribute that allows an arrowhead to be drawn at the end point. Depending on the randomized variable, the start and end point change their positions hence the position of the arrowhead also changes. This randomized variable can then be used in the final equation variable TAns as a factor before the 'U1'. 
Figure 7. Usage of Random Variables in the Model Answer

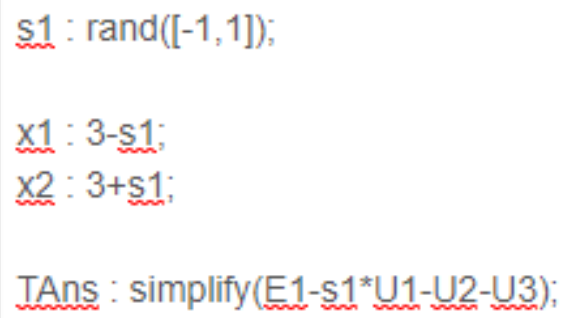

With this method every other voltage arrow in the circuit could also be randomized e.g. with other variables like 's2', 's3', etc. These variables could then be implemented to the final answer to form a correct model answer. Possible programming errors in the solution are relatively easy to fix since they are only dependent on the sign before the factor. The 'simplify' command removes the factors when the answer is displayed for the student making it more readable and understandable.

Due to this simple solution the generation of more complex circuits is possible within a reasonable time frame. A circuit displayed in Figure 8 consisting of three loops for example was used in Circuit Analysis A to teach the students to write the related Kirchhoff's and Ohm's law equations correctly. Every component's direction in this task is randomized which makes the blind guessing basically impossible. Due to the large number of randomized elements in the circuit many different versions can be generated, so the student usually does not get the exact same circuit again. With the method explained earlier the creator only has to type in one equation per input field to cover all the possible solutions of different versions. There is one tricky part, however. The resistor R9 can also be randomized to show a voltage source. In this case the voltage arrow would not be called U9 but E3 instead. This complicates the generation of the answer a little bit since the use of factors is not enough anymore. In this case the random component only affects the inputs of the Loop $\mathrm{C}$ which means we only need to change these model answers accordingly. A simple use of if-else statement is enough to solve the problem. With the use of this method, randomizing the components can make the modelling of the correct answer more complex so one has to think the design of the question carefully through. 
Figure 8. A Circuit with Three Loops used in Circuit Analysis A

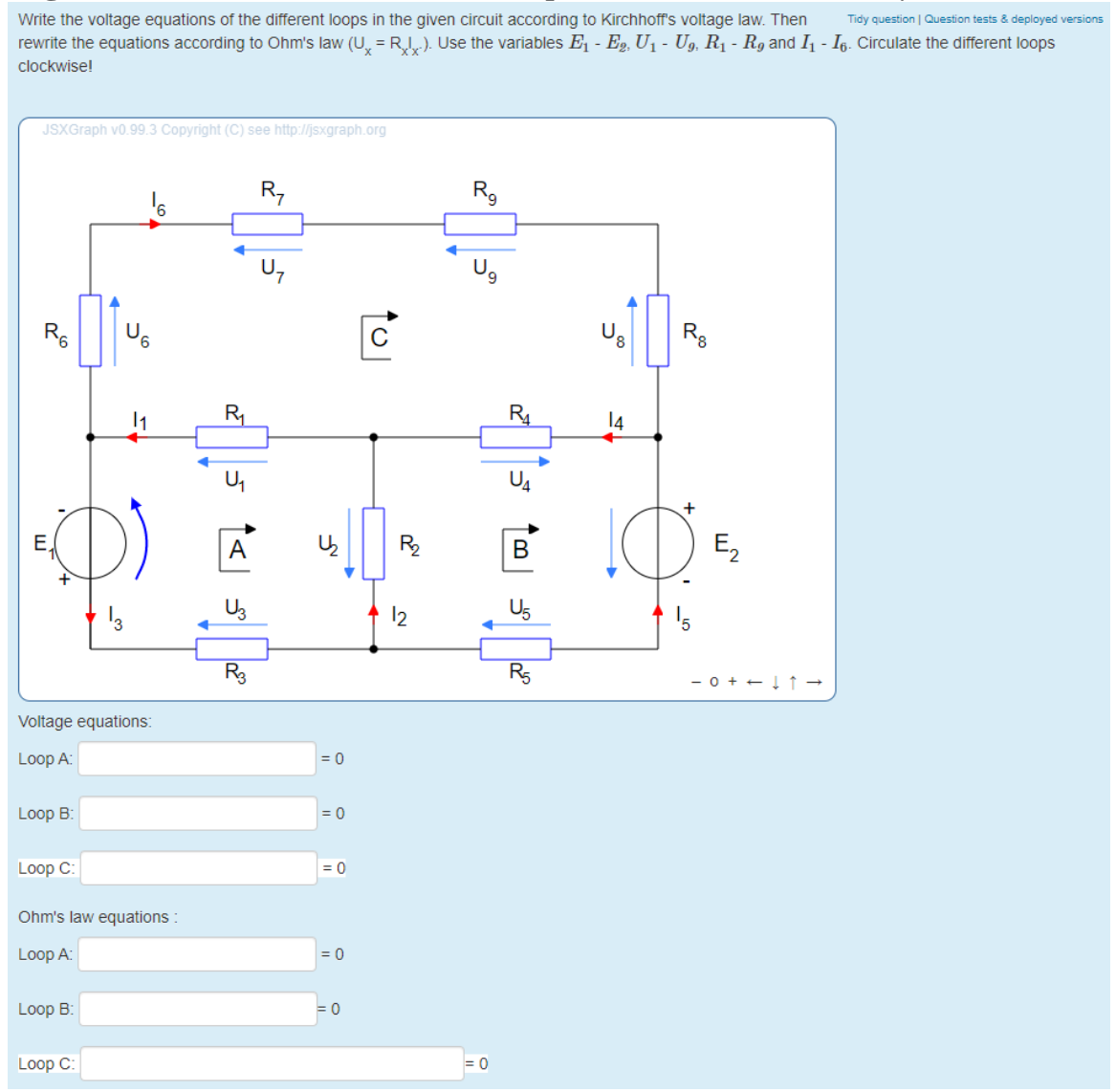

Source: Circuit Analysis A (University of Vaasa 2020).

\section{Various Question Types}

Many different types of questions can be created by using STACK. Traditional types include calculating and formulating equations as shown before. According to some feedback given by the students this can sometimes be frustrating since the typing of the answer has to be exact. One small mistake in the equation makes the whole answer wrong and the student must start from the beginning. This problem becomes more severe with longer and more complex equations with division and brackets. That is why it is also possible to generate more interactive problems without a shown input field. Instead the students can move the elements in picture to right places to give a correct answer. These types of questions can be used for example in vector analysis, where the student is asked to draw a given vector. These types can also be applied in circuit theory.

With the use of 'slider' element some values can be adjusted by the students. Considering a classic problem of reactive power compensation in an AC parallel circuit the student could be asked to set the values of inductance and capacitance of the components so that the reactive power in the circuit is compensated. This setup is shown in Figure 9. With the addition of showing the waveforms of the voltage and current in the circuit and the power triangle and the reactive power generated by the inductor and capacitor, the students should be able to compensate the circuit without calculating anything, thus understanding what compensation 
actually means. The waveforms and the power triangle are calculated from the values of the sliders. This means they change when the sliders are adjusted. Because the sliders are not in practice exact enough, a small error offset is allowed.

Figure 9. An Interactive Question Type, where the Student Adjusts the Sliders to Achieve Full Compensation of the Reactive Power in the Circuit. No Calculation is needed
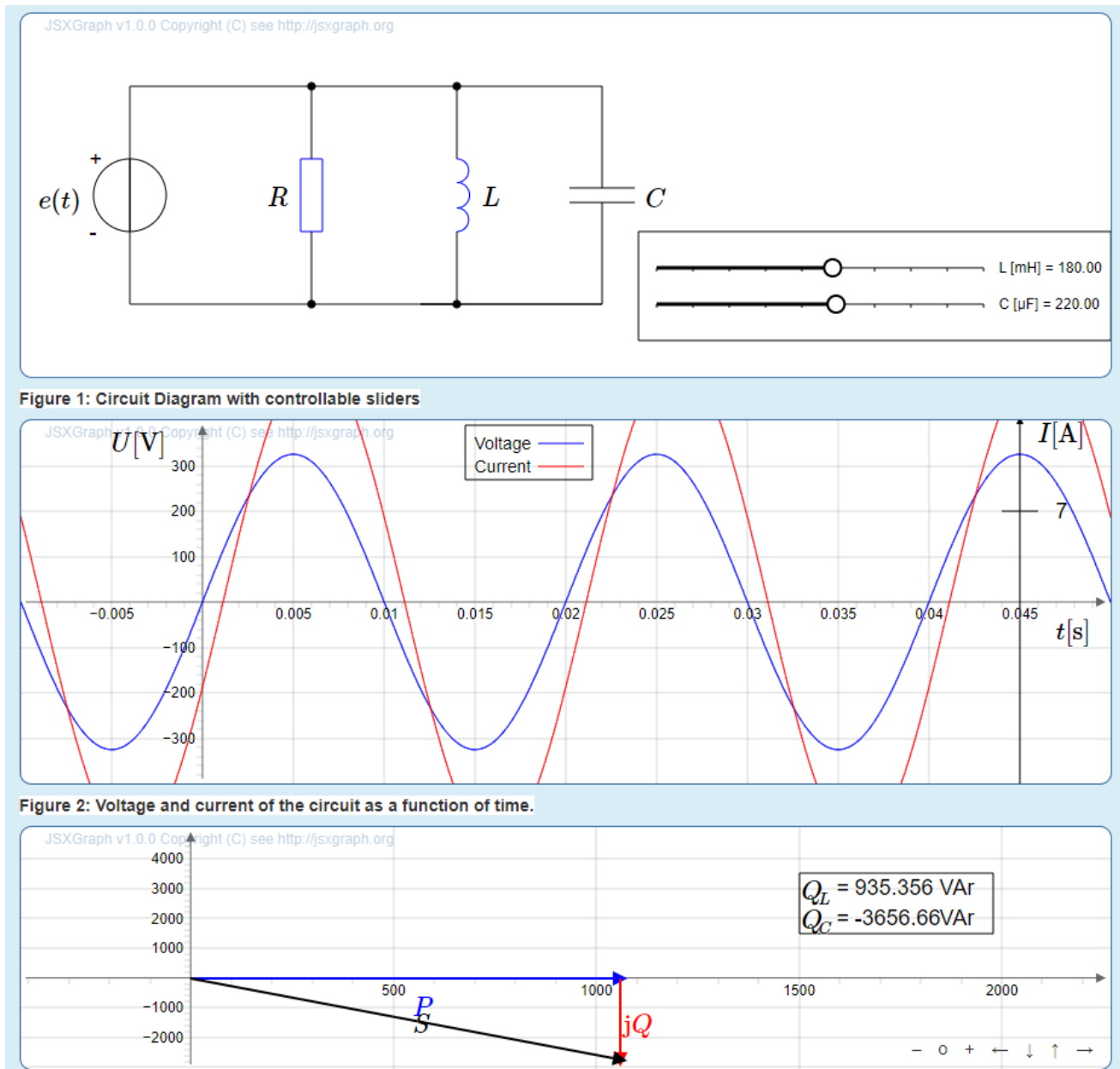

Figure 3: Power Triangle and reactive powers.

In another example the student is prompted to move correct electrical components to correct positions according to the information given in the assignment. This once again does not include any calculation and tests the student ability to understand concepts related to circuit theory rather than mathematical skills. An example of this kind of a question type is presented in Figure 10. The assignment provides the user with information related to the given circuit e.g., equations. The students can then drag and drop different electrical components to right places to satisfy the given equations. The question checks the components' positions and if the student has for example put two components in the same place, an error is given during the evaluation of the answers. This prevents the student from 'cheating' with putting multiple components in the same place hoping that one of them is correct. 
Figure 10. An Interactive Question Type, where the Students are asked to Satisfy the Given Equations by Moving the Correct Components to Correct Positions in the Circuit

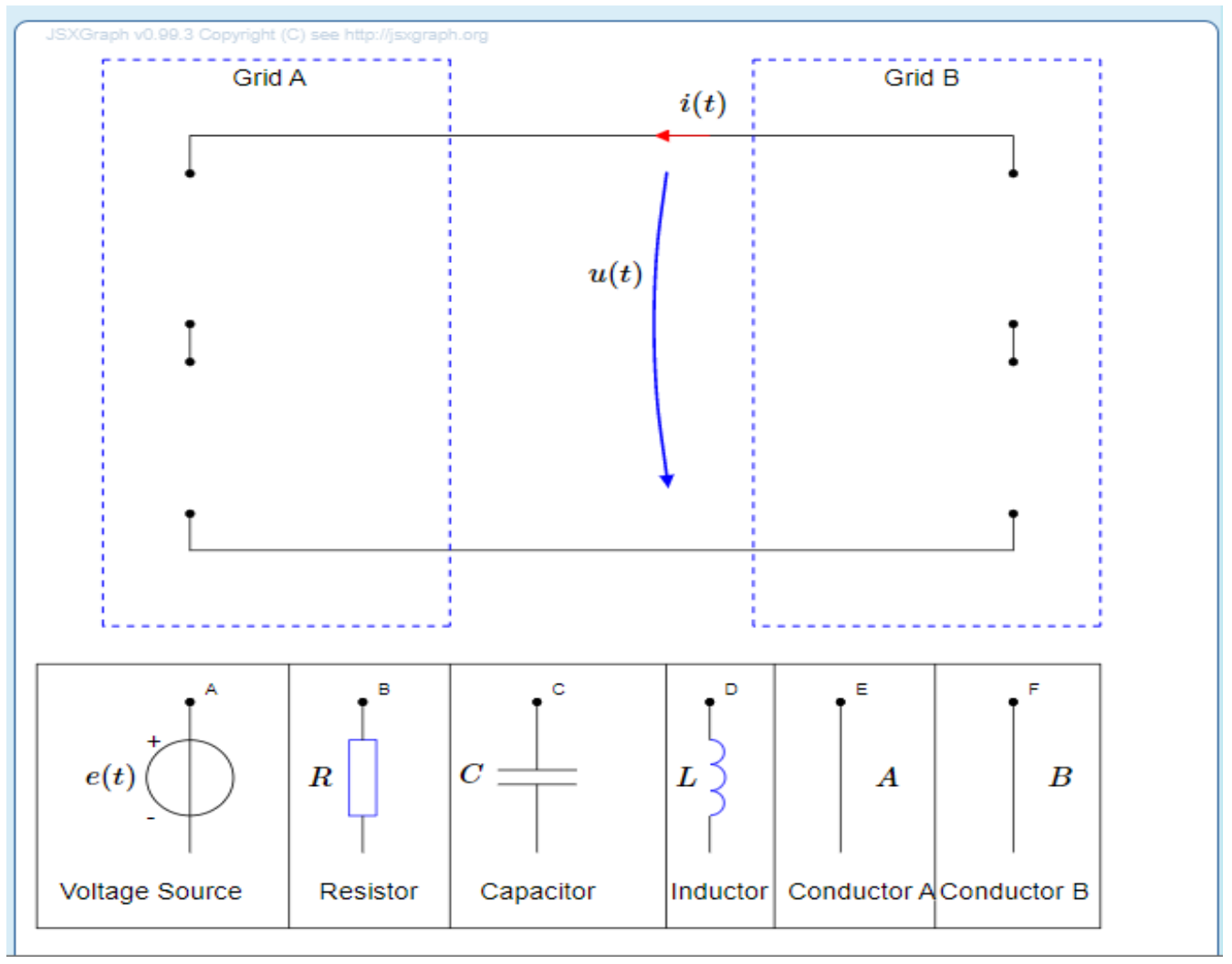

To fully increase the chances for the students to learn from these questions, a complex PRT must be produced. The PRT for the above question for example, consists of 22 nodes which check different things related to the question. A couple of these nodes check the positions of the components and detect if the components overlap each other. Others just check if the answer is correct or not. Some nodes might give the student a special feedback if a known mistake has occurred. This might be the case when the student tries to calculate the apparent power with the given voltage and current. Apparent power is calculated with the following formula:

$$
S=U \cdot I^{*}=|S|<\varphi
$$

where $U$ is the voltage and $I^{*}$ the complex conjugate of the current. A common error made by the students is to leave out the conjugate. This prompts the PRT to give the student a feedback where it is explained, why the answer they gave is wrong and what should be done in order to calculate the correct answer. Because the values for the current and voltage are randomized many versions of the question may be generated and used for training. 


\section{Implementation of STACK Problems in Circuit Analysis}

This section covers how the different problems created with STACK were implemented and used in Circuit Analysis A. As explained earlier the idea of the STACK questions is to help the students understand the basics of each topic so that they could solve the more complex problems introduced in the traditional exercises. This was done in the form of Moodle quizzes. A Moodle quiz can consist of given number of STACK questions. The most used number of questions in a quiz was three. The idea was to make the students to perform the whole quiz repeatedly until they got everything right. Because of this the questions were rather simple and with the more complex problems i.e. problem introduced in Fig. 8, the number of questions inside a quiz was decreased to a minimum of one. This made it possible for the students to learn the necessary skills right after the lectures and before attempting to solve the more complex exercises without spending too much time doing the STACK questions.

At the start of the course all the quizzes were hidden from the students and they were only revealed after the related topics were discussed in the lectures. It was thought that students might react better with more positive attitude when only a few of the quizzes were shown at a time. The students could perform the quizzes repeatedly as many times as they liked without the need to worry about lowering their score since only the best attempt was considered. The questions in the quizzes were presented one at a time. After all the questions were answered the feedback from each of the questions with the student's own answer was given. This made it possible for the student to examine and compare their answers to the right ones or the special feedback.

During the course, the students were asked to immediately contact the lecturer or the creator responsible for the STACK questions in case they noticed some programming errors or other technical problems. This worked quite well, and the questions were usually fixed in a matter of couple of hours. Because of the idea that the students should train with the STACK questions before attending the exercises, deadlines were also issued, although some of the students solved the questions right after they were released and most finished them just before a given deadline. The traditional and the interactive question types worked well apart from programming errors, and there were little to no technical problems associated when the students performed the quizzes.

From the designer point of view there is much to think about when creating this kind of STACK questions. When the STACK question production was started for Circuit Analysis in late 2018, it took very much time to get something done due to number of factors. Our small development team was not sure what kind of possibilities and limits STACK had. This led us to explore and experiment in various ways of visualizing the circuit diagram and later we settled on the JSXGraph presentation which proved to be a good choice. The generation of such complex questions can be time consuming because they need to achieve a certain quality before introduced in the course. This means that the PRT for example, must be on point and must give the students the feedback they need in the best way possible. Without the years of experience, it could be hard to see and think which kind of problems the student might have and what kind of errors they would 
make. Enabling the use of teamwork and fusing together different knowledge and skills made the process possible and quicker.

\section{Feedback Given by the Students}

After the final lectures and exercises on 28.04.2020 an optional feedback from the students was gathered. Out of 25 students, 14 answered a series of questions regarding the STACK problems and issues used in the course $(\mathrm{N}=14)$. The typical questions prompted the users to evaluate the accuracy of given statements with a range from 1 to 6 with 1 meaning 'completely disagree' and 6 meaning 'completely agree'. The two final questions were free word questions asking the students to give some positive feedback and something that could have been better or could be changed. All the statements in the feedback had to be answered.

As seen in Figure 11, when the students were asked if there were too many STACK questions, almost everyone agreed that there were not too many questions. 25 Moodle-quizzes each with 1-6 questions were presented for the students during the course.

Figure 11. One of the Statements in the Feedback

There were too many questions

14 answer

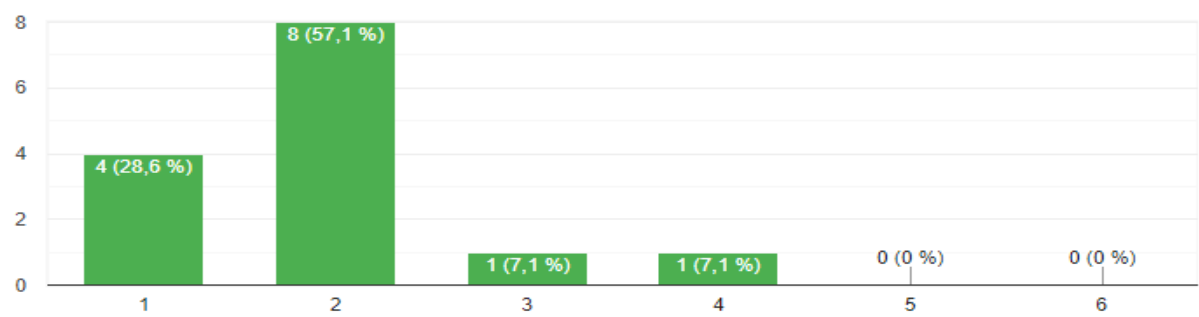

From Figure 12 it can be gathered that the assignments of the given questions were usually clear and understandable.

Figure 12. One of the Statements in the Feedback

The instructions in the assignment were clear

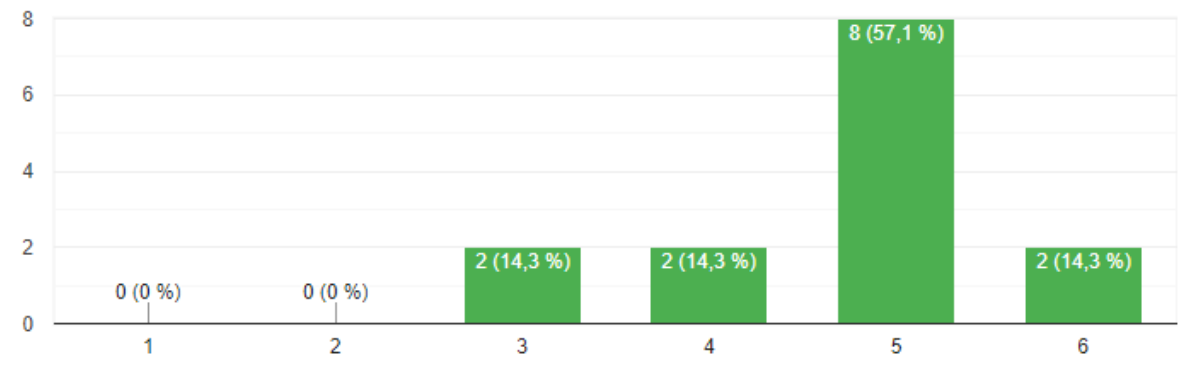


From Figure 13 it can be read that most of the students thought the STACK problems helped them in learning the matters in related topics.

Figure 13. One of the Statements in the Feedback

The questions helped me in learningn the concepts

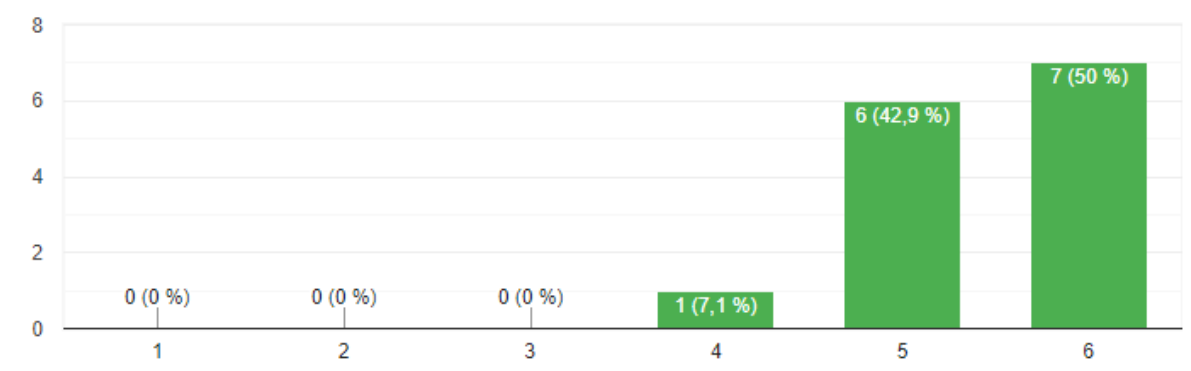

When asked if the problems were too hard to solve, most of the students disagreed with the statement as can be seen in Figure 14.

Figure 14. One of the Statements in the Feedback

The questions were too hard

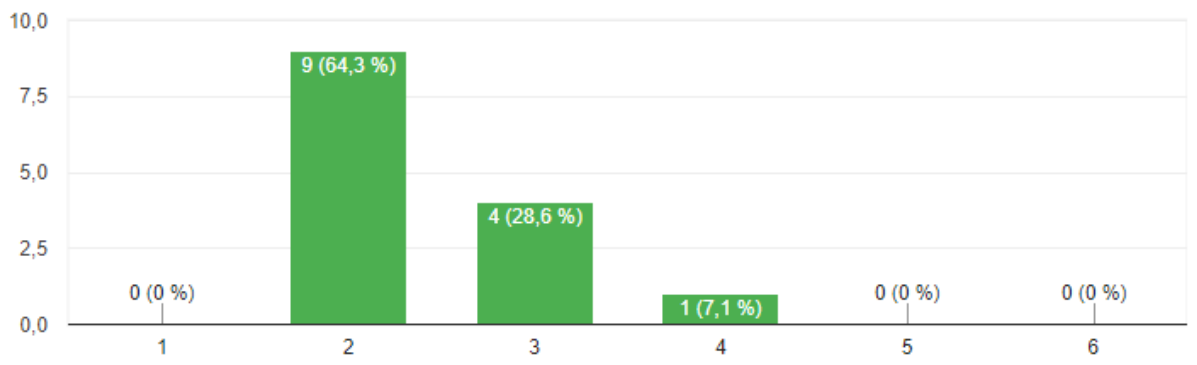

Students also answered a couple of free word questions. Some of the positive feedback included:

- "With the help of the problems, I learned to solve the exercises as well."

- "Simple enough, one could learn the new topics from them."

- "My calculation routine developed."

- $\quad$ The questions helped me to understand some of the concepts better like the Nodal analysis or voltage differences over a component."

- " "Problems helped to illustrate some of the concepts."

Some of the negative feedback with improvement ideas included:

- $\quad$ There could have been more problems. Some of the deadlines could have been given immediately. Now they came out too suddenly." 
- "The feedback from the questions could have been better in some parts."

- "Change the needed percentage for the question to be considered done right from $100 \%$ to $98 \%$. It was frustrating doing a problem for 45 minutes just to begin from scratch due to a miss click."

- "Sometimes it is frustrating to start from scratch if for example a minus sign inside a matrix is missing."

- "There could have been more problems."

This was the first time STACK questions were used primarily as a course material in Circuit Analysis A. In 2019 some of these problems were introduced but more in an experimental manner. Because of this new adaption of STACK there might have been a few problems where the information regarding for example the deadlines, did not come out clearly for the students. As mentioned before, some of the problems had couple of coding errors in there, which have been fixed by now. For the next Circuit Analysis A course, these issues will be fixed.

\section{Conclusions}

The aim of this paper was to introduce the experiences we at the University of Vaasa went through during the development of problems in STACK in the subject of circuit theory. The aim was to find solutions by using STACK for the student's problems related to learning various topics in circuit theory. The questions mentioned in this paper were created for the purpose of enhancing the student's abilities to produce the basic equations of the given circuits in order to better understand the fundamental basics of circuit theory.

During the development process we successfully implemented the use of JSXGraph in order to display circuit schematics and related visual aid in the problems for the students. The randomly generated schematics are relatively easy to produce and according to the feedback gathered by the students they were also easily solvable with no huge problems. The simple method introduced for generating the correct model answer solved the issue that generating bigger circuits would have taken too much time.

The feedback given by the PRT is a very important key element when the students are trying out the skills they have learned during the lectures. Some of the misinformation or missing information can be corrected with the feedback if the error the student made is recognizable. Therefore, extra attention to detail and carefulness should be included when generating the PRT. This prompts the developer to guess beforehand what kind of errors could possibly be made. With the help of years of experience from lecturer Maarit Vesapuisto, we could forecast a couple of the mistakes. Some of the problems were also tailored in such way that they focused on the difficulties the students usually have with the topics.

According to the feedback the problems generated were quite successful. Most of the students thought they were easy enough to understand and solve but also helped them to realize some of the key concepts in given topics. The limitless number of attempts made it possible for some to understand the basics that are 
definitely needed when trying to solve the more complex problems. Naturally, according to the feedback something needs to be improved. For example, in some topics the feedback given by the PRT should have been more detailed according to the students.

From the designer point of view the creation of more unique types of problems might be time consuming. But when we look at the feedback, it can be perceived that all the time spent in planning, coding and constructing these problems definitely pays off. In the future more STACK problems will be constructed in the field of circuit theory. The aim is to create enough problems for the more advanced course of Circuit Analysis B and possibly for other courses too depending on the resources available. The precise presentation of the problems for the students must also be thought thoroughly for the next course in 2021.

More complex engineering problems for example in circuit theory can also be made in STACK. However, in this case presenting the whole problem at once could be very intimidating for the student. In this case it might be wise to introduce the problem for the student piece by piece. With the newer versions of STACK, it is possible to build such problems relatively easy. In the case of complex problems special instructions or tutorials can be given for the students.

\section{References}

Analog Devices (2020) LTspice. Retrieved from: https://bit.ly/3f5sZha.

Attia J (1995) Teaching AC circuit analysis with MATLAB. In IEEE 1995, Proceedings Frontiers in Education $199525^{\text {th }}$ Annual Conference. Engineering Education for the $21^{\text {st }}$ Century. DOI=https://www.doi.org/10.1109/FIE.1995.483086.

Cadence (2020) AWR-APLAC. Retrieved from: https://www.awr.com/.

CircuitLab (2020) Circuit simulation and schematics. Retrieved from: https://www.circuit lab.com/.

EveryCircuit (2020) Circuits are better animated. Retrieved from: https://everycircuit. com/\#0.

Fino M (2018) Challenges in teaching electrical circuit analysis to millennials. In IEEE 2018, $3^{\text {rd }}$ International Conference of the Portuguese Society for Engineering Education (CISPEE). DOI=https://www.doi.org/10.1109/CISPEE.2018.8593460.

JSXGraph (2020) Dynamic mathematics with JavaScript. Retrieved from: https://jsxgra ph.uni-bayreuth.de/wp/index.html.

Maxima (2020) Maxima, a computer algebra system. Retrieved from: http://maxima.sour ceforge.net/.

McDermott L, Shaffer P (1992) Research as a guide for curriculum development: an example from introductory electricity - Part I: investigation of student understanding. American Journal of Physics 60(11): 994-1003.

Moodle (2020) Empower educators with a flexible, open source LMS. Retrieved from: https://moodle.com/.

Neitola M (2019) Circuit theory e-assessment realized in an open-source learning environment. International Journal of Engineering Pedagogy 9(1): 4-18.

Sangwin C (2013) Computer aided assessment of mathematics. New York, USA: Oxford University Press.

STACK Documentation (2018) STACK documentation. Retrieved from: https://stack2. maths.ed.ac.uk/demo2018/question/type/stack/doc/doc.php. 
Trajković L (2011) Teaching circuits to new generations of engineers. In IEEE 2011, International Symposium of Circuits and Systems (ISCAS). DOI=https://www.doi. org/10.1109/ISCAS.2011.5937781.

University of Vaasa (2020) Electrical engineering program description for 2019-2020. Retrieved from: https://bit.ly/38zC3bG.

Valtonen M (2020) Piirianalyysi 1. (Circuit analysis). Finland: Aalto University.

Vesapuisto M (2004) Virtapiirimerkintöjen johdonmukaisuus opetuksen kannalta. (Consistency of electrical circuit markings from the teaching point of view). Master Thesis. Finland: University of Vaasa.

Voipio E (2006) Virtapiirit ja verkot. (Electric circuits and networks). Finland: Otatieto.

WolframAlpha (2020) WolframAlpha, electrical circuit calculators. Retrieved from: https://bit.ly/2AFQMWa. 